\title{
Postoperative infections after dental implant placement: Variables associated with increased risk of failure
}

\author{
Octavi Camps-Font ${ }^{1} \quad$ Pablo Martín-Fatás ${ }^{1} \quad$ Adrià Clé-Ovejero ${ }^{1} \quad$ Rui Figueiredo ${ }^{1}$ | \\ Cosme Gay-Escoda ${ }^{1,2} \quad$ Eduard Valmaseda-Castellón ${ }^{1}$
}

\author{
${ }^{1}$ Oral Surgery and Implantology, Faculty of \\ Medicine and Health Sciences, University of \\ Barcelona, Barcelona, Spain \\ ${ }^{2}$ Oral Surgery and Implantology, Faculty of \\ Medicine, Efhre International University, \\ Belize City, Belize

\section{Correspondence} \\ Rui Figueiredo, Faculty of Medicine and \\ Health Sciences, University of Barcelona, \\ Barcelona, Spain. \\ Email: ruipfigueiredo@hotmail.com
}

\author{
Abstract \\ Background: Wound infections after dental implant placement are a rare finding that \\ might lead to early implant failure. However, the available information on this topic \\ is scarce.
}

Methods: This retrospective cohort study was conducted to determine factors that may increase the failure rate of dental implants that presented a postoperative infection during the osseointegration period. Postoperative infections were defined as the presence of pus or fistula in the surgical area, with pain or tenderness, swelling, redness, and heat or fever, before prosthetic loading. A bivariate and multivariate analysis of the data using Cox proportional-hazards regression was performed to detect prognostic factors for implant failure in patients that suffer infections.

Results: The patient-based prevalence of postoperative infections after implant placement was $2.80 \%$ (95\% confidence interval (95\%CI): $2.04 \%$ to $3.83 \%$ ). Thirty-three out of $37(89.19 \%)$ patients with infections had to be surgically retreated because of antibiotic failure and $65 \%$ of the infected implants were removed. The bivariate analysis showed a significant association between implant failure and the collar surface (HR: 3.12 ; $95 \% \mathrm{CI}$ : 1.16 to $8.41 ; P=0.014$ ). Cox proportional-hazards regression indicated that rough-surfaced collars increased 2.35 times the likelihood of failure (95\% CI: 0.87 to $6.37 ; P=0.071)$.

Conclusions: The survival of implants placed in the maxilla, with smooth collar, and late-onset of infection was higher than those placed in the mandible, with a rough collar and early onset of infection. In general, signs of infection after dental implant placement compromises the survival rate of the affected fixtures.

\section{K E Y W O R D S}

dental implants, postoperative complications, prognosis, surgical wound infection, survival analysis

\section{1 | INTRODUCTION}

Over recent decades, the use of dental implants for oral rehabilitation has been proven to be a reliable therapeutic option in a broad variety of scenarios, due to its positive long-term clinical results. ${ }^{1,2}$ However, both short- and long-term complications may arise. ${ }^{3}$
Postoperative infections are considered a rare complication, with a prevalence ranging from $1.6 \%$ to $11.5 \% .^{4-12}$ They usually occur within the first month after dental implant placement. ${ }^{4}$ Changes in the oral flora caused by postoperative short-duration systemic and topical antibacterial therapy could also favor the development of opportunistic infections. Nonetheless, as with any biomaterial-centered infection, such 
complications do not respond to antibiotics and frequently persist until the implant is removed. ${ }^{13,14}$ Accordingly, postoperative infections have been suggested as a risk factor for osseointegration, ${ }^{15,16}$ increasing the risk of early failure almost 80 -fold. ${ }^{17}$

Patients undergoing dental implant placement in the mandible with submerged healing seem to be more prone to develop these postoperative infections. ${ }^{17}$ However, very limited information is available on the survival of infected implants and on the main prognostic factors for early failure. Therefore, the aim of the present study was to determine factors that may increase the failure rate of dental implants that have presented a postoperative infection during the osseointegration period.

\section{2 | MATERIALS AND METHODS}

A retrospective cohort study was conducted in a total of 1,322 outpatients $(2,673$ implants) who were treated consecutively between January 2004 and October 2015 through the Oral Surgery and Implantology Master's degree program of the University of Barcelona. The study design followed the STROBE guidelines for cohort studies. ${ }^{18}$ The protocol complied with the Helsinki Declaration and was approved by the Ethical Committee for Clinical Research (CEIC) of the Dental Hospital of the University of Barcelona (Spain).

Patients were given full information about the surgical procedures and treatment alternatives, and informed consent was obtained in all cases. The preoperative analysis included clinical and radiographic examinations (with panoramic radiographs or computed tomography).

The exclusion criteria were general contraindications to implant surgery, such as an American Society of Anesthesiologists (ASA) health status score ${ }^{19}$ higher than 3, immunosuppression, bleeding disorders, active treatment of malignancy, drug abuse, psychiatric illness, and intravenous bisphosphonate use. ${ }^{20,21}$ Patients under 18 years of age were also excluded.

Patients with active periodontal disease were treated prior to the study in accordance with the American Academy of Periodontology guidelines. ${ }^{22}$

Postoperative infections were defined as the presence of purulent drainage (pus) or a fistula in the operated region, with pain or tenderness, localized swelling, redness, or heat or fever $\left(>38^{\circ} \mathrm{C}\right)$, before prosthetic loading. ${ }^{23}$ If the postoperative infection involved several implants, then one of them was selected using a random-numbers table based on the position of the implant according to the double-digit FDI World Dental Federation classification.

To avoid misclassification bias, any of the following circumstances were excluded from the analysis: incomplete clinical records, dental implants placed in previous failure sites, patients who required guided bone regeneration procedures or procedures involving non-conventional prosthetic loading (the inclusion criteria were $\geq 3$ months in the mandible and 4 months in the maxilla after implant placement).

Early implant failure was defined as the absence or removal of the dental implant at any point in time after its placement and before the final restoration was placed. ${ }^{16}$

The patients' records were followed up through to prosthetic loading. The follow-up time was defined as the time from placement to failure or to prosthetic abutment connection, whichever happened first.

\section{1 | Surgical procedure}

Implants were placed under local anesthesia, generally with articaine in a $4 \%$ solution with epinephrine $1: 100,000,{ }^{*}$ by third-year fellows of the Master's degree program in Oral Surgery and Implantology. The surgical technique employed has been described thoroughly in a previous report. ${ }^{4}$ According to our protocol, implants rough surface was totally covered by bone. If the insertion torque was higher than $15 \mathrm{~N} \cdot \mathrm{cm}^{2}$, a healing abutment was placed. The flaps were usually repositioned with 4-0 polyamide ${ }^{\dagger}$ sutures. The suture was removed 7 to 15 days after surgery.

After the operation, an antibiotic (usually amoxicillin $750 \mathrm{mg}^{\ddagger}$ orally every 8 hours for 7 days), a non-steroidal antiinflammatory drug (usually ibuprofen $600 \mathrm{mg}^{\S}$ orally every 8 hours for 4 to 5 days), an analgesic (usually paracetamol $1 \mathrm{~g}^{\text {II }}$ orally every 8 hours for 3 to 4 days), and a mouthrinse ( $0.12 \%$ chlorhexidine digluconate ${ }^{\#} 15 \mathrm{~mL}$ every 12 hours for 15 days) were prescribed.

Postoperative and prescribed drug use instructions were explained and were handed to the patient on a sheet of paper. Patient compliance was not specifically assessed.

\subsection{Treatment of the postoperative infection}

After infection onset, an antibiotic was prescribed for 7 days (usually amoxicillin with potassium clavulanate or clindamycin) and the patients were instructed to perform chlorhexidine digluconate mouthrinses. If this therapy was insufficient to control the infection, another antimicrobial was prescribed and a surgical procedure consisting in implant removal (if the implant had mobility or advance bone loss), second stage surgery or mechanical debridement with plastic curets was performed.

\footnotetext{
* Artinibsa; Inibsa Dental, Lliçà de Vall, Spain

$\dagger$ Supramid; Aragó, Barcelona, Spain

†Clamoxyl; GlaxoSmithKline, Madrid, Spain

$\S$ Algiasdin; Esteve, Barcelona, Spain

II Gelocatil; Gelos, Barcelona, Spain

* Clorhexidina Lacer; Lacer, Barcelona, Spain
} 


\section{3 | Data sampling}

A single trained researcher (OCF) examined all the clinical records. The following data were retrieved: date of birth, gender, patient health status based on the ASA Physical Status Classification System (ASA category 1 or ASA category >1), ${ }^{19}$ smoking habit (non-smoker or smoker), periodontal disease (healthy or periodontally compromised), implant manufacturer, implant surface (non-anodized or anodized), implant collar (smooth or rough surface), location (maxilla or mandible), position (anterior or posterior), implant placement timing ( $\leq 8$ or $>8$ weeks after tooth extraction), primary stability (insertion torque higher than $15 \mathrm{~N} \cdot \mathrm{cm}^{2}$ ), submerged or non-submerged healing, surgeon who performed the operation and the postoperative follow-up appointments, and final outcome (survival or early failure). After infection diagnosis, additional data were also recorded: antibiotic prescribed, treatment duration, and need for an additional surgical procedure (none, surgical debridement, second stage surgery or implant removal). The dates of implant placement, infection diagnosis, infection resolution, and last follow-up were also recorded.

\section{4 | Statistical analysis}

The sample size calculation was based on the assumptions that $54.5 \%$ (SD: 0.50) of postoperative infections lead to implant failure ${ }^{4}$ and that the development of infection in implants with a rough-surfaced collar increases the risk of early implant failure at least 3-fold. Considering a coefficient of determination $\left(\mathrm{R}^{2}\right)$ of 0.25 (implant collar surface variable), an $\alpha$ risk of 0.05 and a power of $80 \%, 35$ patients with an infection were required.

The statistical analysis was carried out with Stata14.*

The subjects' characteristics were presented as absolute and relative frequencies for categorical outcomes. Normality of scale variables (patient age and time from implant placement to infection onset) was explored using the Shapiro-Wilk test and through the visual analysis of the P-P plot and box plot. Where normality was rejected, the interquartile range (IQR) and median were calculated. Where distribution was compatible with normality, the mean and standard deviation (SD) were used.

Cumulative survival rates were calculated using the Kaplan-Meier method. Univariate analyses using the log-rank and univariate Cox proportional-hazards regression for continuous variables were performed to identify the association between each categorical and continuous covariate with implant survival, respectively. Hazard functions (h) and hazard ratios (HR) with $95 \%$ confidence intervals $(95 \% \mathrm{CI})$ were calculated for each covariate. Odds ratio (OR) with 95\% CI

\footnotetext{
* StataCorp, College Station, TX
}

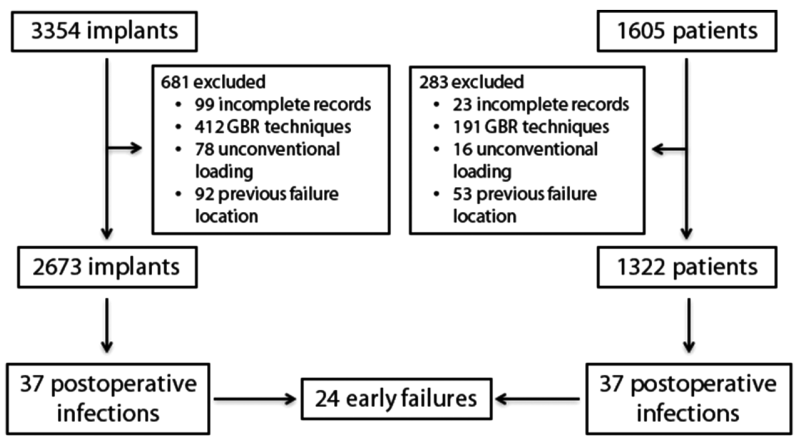

F I G URE 1 Flowchart of participants in the present study

was estimated to determine the influence of periodontal status on the risk of postoperative infection, selecting randomly one control (i.e. patients who underwent the same surgical procedure within the above-mentioned time frame, but did not develop infection during the postoperative period) for each infected patient. The level of significance was set at $P<0.05$.

A multivariate analysis was performed using a Cox proportional-hazards model to assess the contribution of each of the predictive variables and to construct a predictive model of postoperative infection resolution. Based on all the possible subsets obtained by combining the maximum model terms (i.e. all independent covariates which had a $P$ value $<0.30$ in the bivariate analysis), the most parsimonious model with the highest maximum predictive power (Harrell's c statistic) was selected. The predictive power of the selected survival model was compared to that of the maximum model to verify that there were no differences between them. ${ }^{24}$ Goodness of fit of the data was assessed by plotting the cumulative hazard function of Cox-Snell residuals. The Cox proportionalhazards regression equation was used to calculate the adjusted HR of the predictive variables with a 95\% CI.

\section{3 | RESULTS}

The 1,322 patients who met the inclusion criteria received 2,673 implants (Figure 1).

Thirty-seven postoperative infections (37 patients) were recorded. The patient- and implant-based prevalence was $2.80 \%$ (95\% CI: $2.04 \%$ to $3.83 \%$ ) and $1.38 \%$ (95\% CI: $1.01 \%$ to $1.90 \%$ ), respectively. Tables 1 and 2 show the main demographic, surgical, and postoperative variables of the sample.

The data were not affected by any loss to follow-up. The study time ranged from 19 to 208 days with a median of 55 days (IQR: 111 days), during which 24 of the infected implants (64.9\%) were removed. Nine patients who overcame the postoperative infection required an additional surgical procedure consisting of second stage surgery (if the implant was submerged: six cases, $16.22 \%$ ) or mechanical debridement with plastic curettes (three cases, $8.11 \%$ ). 
TA B L E 1 Results of the categorical variables

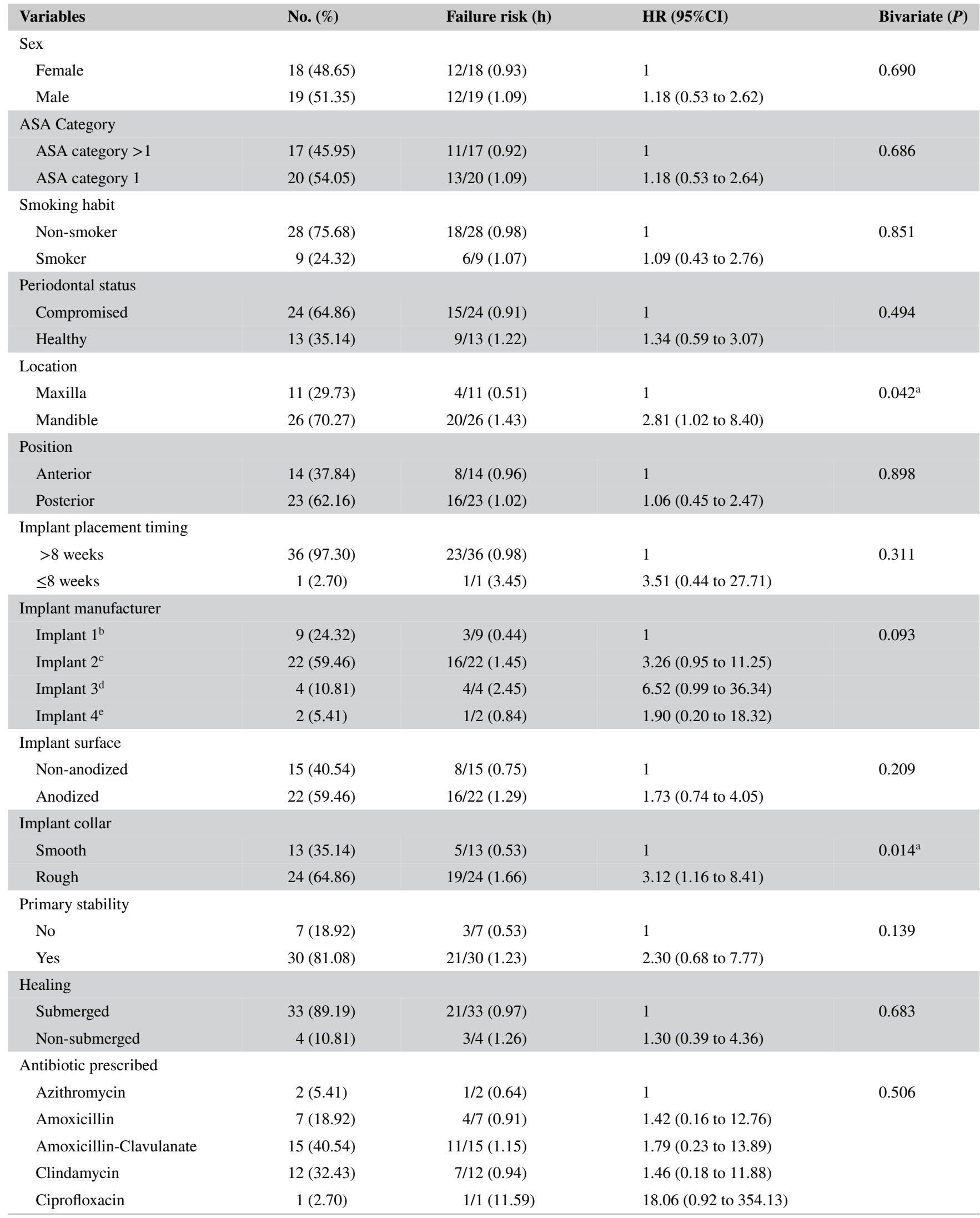

${ }^{a}$ In the bivariate analysis, mandible location and rough implant collar were significantly associated with infections $(P<0.05)$.

${ }^{b}$ Phibo, Sentmenat, Spain.

${ }^{\mathrm{c}}$ Nobel Biocare, Kloten, Switzerland.

${ }^{\mathrm{d} D E N T S P L Y ~ I m p l a n t s, ~ M o ̈ l n d a l, ~ S w e d e n . ~}$

${ }^{\text {e}}$ Straumann AG, Basel, Switzerland. 
T A B L E 2 Results of the scale variables

\begin{tabular}{|c|c|c|c|}
\hline Variables & $\begin{array}{l}\text { Mean (SD) or } \\
\text { median (IQR) }\end{array}$ & HR (95\% CI) & $\begin{array}{l}\text { Bivariate } \\
(P)\end{array}$ \\
\hline Age & $56.50(12.53)^{b}$ & 0.97 (0.94 to 1.00$)$ & 0.081 \\
\hline $\begin{array}{l}\text { Time from implant } \\
\text { to infection } \\
\text { (weeks) }\end{array}$ & $4.14(4.43)^{\mathrm{c}}$ & $1.12(1.02$ to 1.22$)$ & $0.005^{\mathrm{a}}$ \\
\hline Antibiotic duration & $10(8)^{\mathrm{c}}$ & $0.93(0.85$ to 1.01$)$ & 0.057 \\
\hline
\end{tabular}

${ }^{a}$ In the bivariate analysis, time from implant placement to infection was significantly associated with infections $(P<0.05)$.

${ }^{\mathrm{b}}$ Mean (SD).

${ }^{\mathrm{c}}$ Median (IQR).

Overall, the cumulative survival rate was $33.45 \%$ (95\% CI: $18.50 \%$ to $49.12 \%$ ) at 208 days after dental implant placement (Figure 2A). Univariate analysis showed a significant association between implant failure and time from implant placement to postoperative infection (HR: 1.12 ; $95 \%$ CI: 1.02 to 1.22 ; $P=0.005$ ), implant collar surface (HR: $3.12 ; 95 \%$ CI: 1.16 to $8.41 ; P=0.014)$ and location of the infected dental implant (HR: $2.81 ; 95 \%$ CI: 1.02 to $8.40 ; P=0.042$ ) (Tables 1 and 2 ). The survival curves for maxillary location, smooth implant collar, and late-onset infection were higher than for their counterpart groups (Figures 2B through 2D).
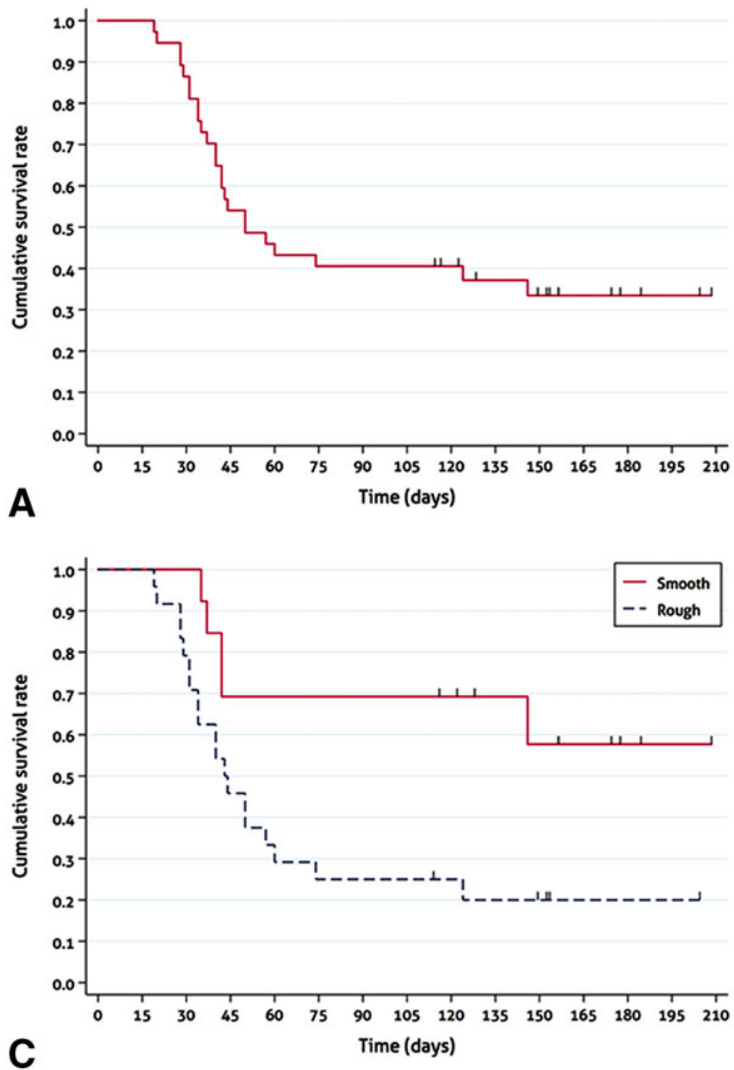

T A B L E 3 Adjusted hazard ratio (HR) of the variables included in the Cox proportional-hazards regression model

\begin{tabular}{|lll|}
\hline Variables & $\begin{array}{l}\text { Adjusted HR } \\
(\mathbf{9 5 \%} \mathbf{C I})\end{array}$ & $\begin{array}{l}\text { Cox } \\
\text { Regression }(\boldsymbol{P})\end{array}$ \\
\hline Rough implant collar & $2.35(0.87$ to 6.37) & 0.071 \\
\hline $\begin{array}{l}\text { Time from implant } \\
\text { to infection }\end{array}$ & $1.11(1.00$ to 1.22) & 0.023 \\
\hline
\end{tabular}

Twenty-four infected patients $(64.86 \%)$ and 21 controls (56.76\%) were periodontally compromised (OR $=1.41 ; 95 \%$ CI, 0.56 to $3.56 ; P=0.475$ ).

The final Cox proportional-hazards regression model included the following independent variables: implant collar surface and time from implant placement to postoperative infection onset (Table 3). Each week earlier in the appearance of postoperative infection multiplied the adjusted risk of failure by 1.11 (95\% CI: 1.00 to $1.22 ; P=0.023$ ). Additionally, dental implants with rough-surfaced collars increased the adjusted risk of failure by 2.35 times compared to those with a smooth collar surface (95\% CI: 0.87 to $6.37 ; P=0.071$ ).

The change in the likelihood ratio of the Cox proportionalhazards regression model was significant $\left(\chi^{2}=11.21 ; \mathrm{df}=2\right.$; $P=0.004)$. Despite the small number of cases, the hazard
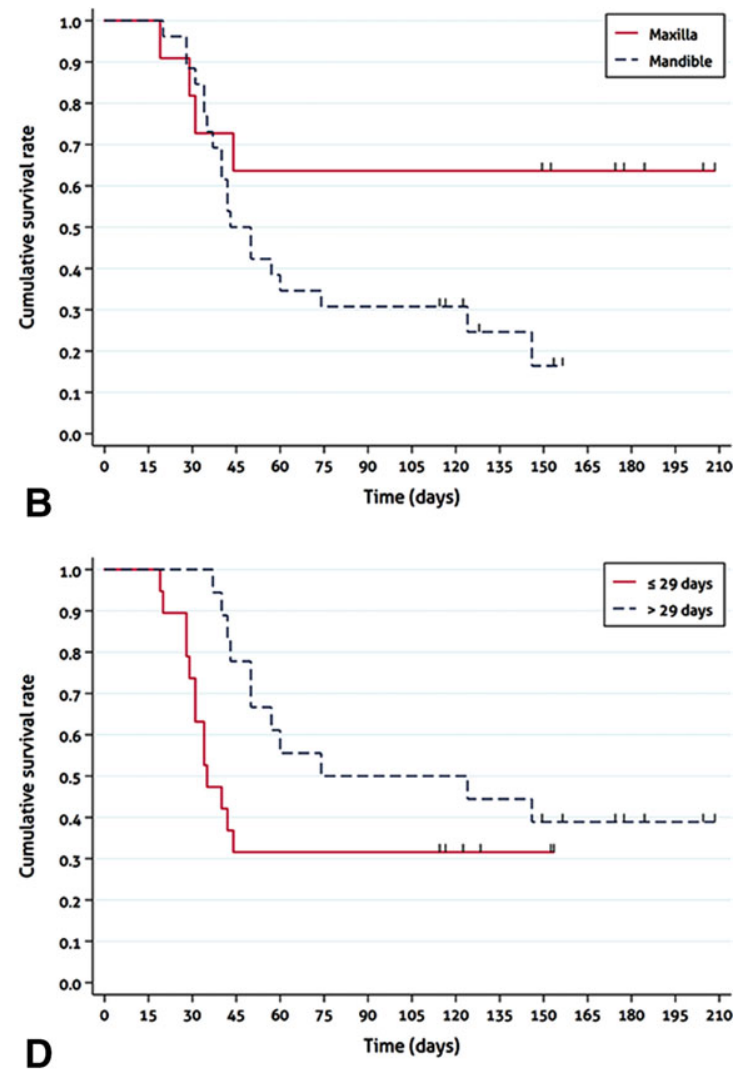

F I G U R E 2 Kaplan-Meier survival curves for implant survival. A) overall outcome, B) by implant location (maxilla/mandible), C) by implant collar surface (smooth/rough) and D) by time from dental implant placement to infection (4 weeks or earlier/over 4 weeks after implant placement). Vertical lines indicate survival from infection, followed by prosthetic loading 


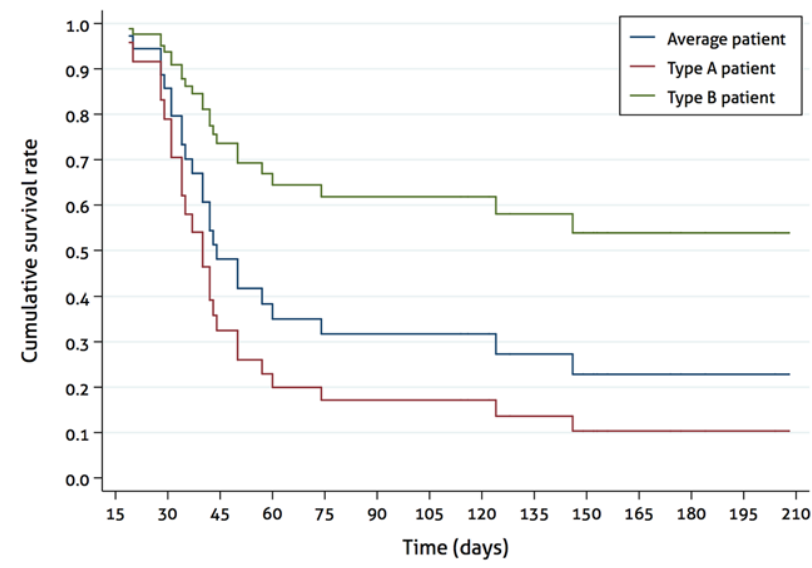

F I G U R E 3 Predicted probability of survival of three hypothetical patients with average, favorable and unfavorable characteristics. Type A (most unfavorable) is a subject with a rough-collared implant who develops an infection 2.9 weeks (the value that defines the first quartile of this variable) after implant placement. Type B (most favorable) is an individual with a smooth-collared implant who develops an infection after 7.3 weeks (the value that defines the third quartile of this variable)

function of Cox-Snell residuals showed a good fit with the data. The assumptions of the model were fulfilled: none of the variables included in the final model had a time-varying effect, either collectively or individually $(P=0.055$ and $P=0.445$ for time from implant placement to postoperative infection onset and implant collar, respectively), thus supporting the assumption of proportional hazard, and there was a log-lineal relation $(P=0.786)$.

Harrell's c statistic was 0.78 (95\% CI: 0.65 to 0.91 ), showing good predictive power. The loss of predictive power between the model selected and the maximal survival model was not significant $(-2.07 \%$; $95 \%$ CI: $-8.99 \%$ to $4.85 \%$; $P=0.538$ ). Figure 3 shows the predicted probability of survival of three hypothetical patients with different patterns of characteristics (i.e. unfavorable, average and favorable cases). Similarly, supplementary Table S1 in the online Journal of Periodontology depicts the predicted probability of survival at the end of the follow-up period (208 days) for several clinical scenarios.

\section{4 | DISCUSSION}

This study aimed to identify some of the prognostic factors for failure in dental implants that presented postoperative infections. Prognostic research provides information to patients about possible outcomes, identifies risk groups for stratified management, and helps target specific prognostic factors for modification. ${ }^{25}$ Several reports have addressed the prevalence and described some of the clinical features and risk factors for postoperative infections. ${ }^{4-12,17}$ However, to the best of the authors' knowledge this cohort study adds useful new information to the literature, since it identifies which patients and implants are associated with a higher risk of failure after a postoperative infection. Thus, this study provides data that might help clinicians to decide whether to treat the infection or to remove the implant directly due to its poor prognosis. It is also interesting to consider a previous report by CampsFont et al. ${ }^{4}$ which shows that loaded implants that have previously suffered a postoperative infection have a poor outcome in the short-term (a success rate of 50\% after a mean follow-up period of 43 months).

The main limitations of the present study are its retrospective nature, which did not allow the recording and analysis of all possible potential prognostic factors. In this sense, future research should address the role of covariates related with the etiopathogenesis of early implant failure such as bone overheating of the surgical site, ${ }^{26}$ bone quality and surgeons' experience. Another limitation of the study is the criteria used to define infection, based mostly on clinical observations. However, few conditions other than infections can be considered in the differential diagnosis of patients who start having pain a few days after implant placement. Some methods, such as determining C-reactive protein levels, could help to detect infections in a more objective way. ${ }^{27}$ Also, it should be interesting to evaluate in the future, if having a preoperative conebeam computed tomography (CBCT) decreases the rate of complications and of postoperative infection. This variable could not be assessed in the present cohort because the criteria used to perform a CBCT before surgery varied throughout the years (after 2011 almost all patients included in the study had a CBCT available, whereas that was not the case for cases included in the initial years of the cohort).

The authors decided to exclude implants that required simultaneous bone grafting techniques because these procedures can increase the risk of infection, especially when membranes are exposed due to inadequate wound closure.

Although the prevalence per patient of postoperative infections when systemic antibiotics are administered varies considerably across published studies, Esposito et al. ${ }^{28}$ reported a weighted rate of $2.3 \%$ (95\% CI: $0.4 \%$ to $4.1 \%$ ). These numbers are quite similar to those reported in this study. In contrast, the small number of participants in some reports, ${ }^{9-12}$ the absence of standardized diagnostic criteria, the different research designs and the diversity of the demographic characteristics of the samples could partially explain the wide range of infection rates.

A single preoperative administration of antibiotics seems to reduce the failure rate of dental implants placed under ordinary conditions. However, this single dose does not seem to have any effect on prevention of postoperative infections. In fact, a recent meta-analysis reported no statistically-significant differences regarding infection prevalence when antibiotics were administered $(5.9 \%$ versus $7.0 \%$. $P=0.39){ }^{28}$ 
The cumulative survival rate is the probability that an implant will survive to a particular point in time. In this study, the number of implants that failed over the osseointegration period numbered 24 out of a total of 37 cases of postoperative infection, yielding a cumulative survival rate of $33.5 \%$ at the end of the follow-up. This finding is in accordance with papers that have considered postoperative infections one of the main risk factors for early implant failure. , $^{5,10,15-17}$

A recent study by the present research group revealed that dental implants placed in the mandible are more prone to infectious complications. ${ }^{17}$ The bivariate analyses performed in the present study also identified a statistically significant association between mandibular location and implant failure (Table 1 and Figure 2D). The reason might be that the relatively poor blood supply and macro- and micro-architecture (thick cortical plates and small medullary spaces) of the mandible might hamper the already complex management of this complication. However, this variable was not included in the final Cox proportional-hazards regression model, as other variables could act as confounders. Indeed, the relationship between implant location and failure varied when stratified by primary stability $\left(\chi^{2}=2.13 ; \mathrm{df}=1 ; P=0.145\right)$, which might suggest that primary stability could be a confounding factor.

The previous history of periodontitis was not related to an increased risk of developing a postoperative infection neither a higher probability of early failure in case of suffering this complication. These findings are consistent with a previous report of the present research group ${ }^{17}$ and could be partially explained because all patients with active periodontal disease were treated before implant placement. ${ }^{22}$

Traditionally, it has been suggested that early signs of infection after dental implant placement may be much more critical than if the same complication occurs later, because the process of osseointegration can be disturbed. ${ }^{29}$ Late superficial postoperative infections of soft tissues are generally uncomplicated and can sometimes be attributed to remnants of suture material, to insufficient tightening of the cover screw or to excessive pressure of the dentures on the underlying mucosa. ${ }^{30}$ The present results agree with previous publications, since late-onset postoperative infections were associated with a better prognosis. Indeed, each week earlier in the appearance of postoperative infection multiplied the adjusted risk of failure by 1.11 . Besides, more than three-quarters of the postoperative infections which led to implant failure occurred during the first 2 months after placement. Nevertheless, the fact that this complication has a delayed onset (it generally occurs 1 month after the surgical procedure), probably due to the postoperative use of antibiotics and antiseptic mouthrinses, highlights the importance of establishing a strict patient follow-up protocol during the first postoperative weeks to initiate early treatment if required.

Generally, rough implant surfaces enhance initial adhesion, attachment, and colonization by bacteria and favor plaque formation. Zaugg et al. ${ }^{31}$ concluded that rougher surfaces increase bacterial adhesion and make biofilm removal more difficult. Consequently, when some degree of bone loss occurs these surfaces might favor the onset and progression of peri-implant diseases. ${ }^{32,33}$ The present results suggest that implants with rough-surfaced collars had a worse prognosis even after pharmacological treatment of the postoperative infection. Indeed, these implants had an adjusted 2.35 times higher probability of early implant failure when compared with smooth-surfaced collar fixtures. In future, prospective studies should be conducted to confirm this relation.

Smoking has been well-established as a risk factor for periodontitis and peri-implant diseases. ${ }^{34,35}$ Moreover, several studies have shown that tobacco reduces implant survival. ${ }^{36-39}$ Surprisingly, this association was not found in the present study. In the authors' opinion, this could be due to the fact that smoking was recorded as a dichotomous variable because of the small number of cases. Moreover, the amount of tobacco was self-reported, leading to possible recall bias.

The impact of systemic diseases on the outcome of implant therapy remains unclear. Although some investigators have reported higher complication rates in medically compromised patients, ${ }^{40}$ others, in accordance with the present observations, have suggested that some conditions do not seem to influence the treatment outcomes. ${ }^{41}$ Nevertheless, it seems reasonable to assume that the severity of systemic diseases can be far more critical than the disorder itself. Therefore, a strict preoperative assessment allowing adequate diagnosis and management of any systemic disorder is mandatory because it can lower the complication rates of the implant therapy.

It has been claimed that some anaerobes can attach directly to an inert titanium surface, colonize it and subsequently lead to infection of the peri-implant tissues. ${ }^{42,43}$ Accordingly, amoxicillin plus potassium clavulanate, clindamycin, or metronidazole should be three of the most suitable antibiotic treatments for this complication. However, none of the antibiotics proved more effective than any of the others in the present sample (Table 1). Indeed, 33 out of 37 (89.19\%) patients with infections had to be surgically retreated because of antibiotic therapy failure. This stresses the importance of performing a study to identify the bacteria involved and their susceptibility to commonly used antibiotics, to determine the most adequate drugs to treat such infections. In addition, when systemic antibiotics are prescribed empirically without microbiological monitoring, the appearance of superinfections and the overgrowth of opportunistic pathogens difficult to eradicate can occur. ${ }^{44}$ Hence, local application of the antibiotics might be specially indicated in this complication, since systemic administration might produce low bioavailability of the drug in the infected region. A more effective therapeutic approach might lead to improvements in the survival and success rates of these implants. 


\section{5 | CONCLUSIONS}

Patients that suffer a postoperative infection after dental implant placement have a poor prognosis, with a cumulative survival rate of $33.5 \%$. Surgical therapy was required to treat $89 \%$ of infections since systemic antibiotic therapy was insufficient in most cases. Implants with a rough-surfaced collar where an early postoperative infection develops seem to be more prone to suffer early failure of the fixture.

\section{ACKNOWLEDGMENTS}

The authors wish to thank Mary Georgina Hardinge (native British freelance translator) for translating the manuscript into English. This study was conducted by the IDIBELL Institute's Dental and Maxillofacial Pathology and Therapeutics research group. Dr. Figueiredo reports grants, personal fees, and non-financial support from MozoGrau (Valladolid, Spain) and personal fees from BioHorizons Ibérica (Madrid, Spain), Inibsa Dental (Lliça de Vall, Spain), DENTSPLY Implants Iberica (Barcelona, Spain) and ADIN Implants (Afula, Israel) outside the submitted work. Dr. Figueiredo has also participated as a principal investigator in a randomized clinical trial sponsored by Mundipharma (Cambridge, UK) and in another clinical trial as a subinvestigator for Menarini Richerche (Florence, Italy). Dr. Valmaseda-Castellón has given lectures for BioHorizons and MozoGrau. The remaining authors report no potential conflicts of interest.

\section{REFERENCES}

1. Jung RE, Pjetursson BE, Glauser R, Zembic A, Zwahlen M, Lang NP. A systematic review of the 5-year survival and complication rates of implant-supported single crowns. Clin Oral Implants Res. 2008;19:119-130.

2. Albrektsson T, Donos N. Implant survival and complications. The Third EAO consensus conference 2012. Clin Oral Implants Res. 2012;23(Suppl. 6):63-65.

3. Derks J, Tomasi C. Peri-implant health and disease. A systematic review of current epidemiology. J Clin Periodontol. 2015;42 (Suppl. 16):158-171.

4. Camps-Font O, Figueiredo R, Valmaseda-Castellón E, Gay-Escoda C. Postoperative infections after dental implant placement. Implant Dent. 2015;24:713-719.

5. Esposito M, Cannizzaro G, Bozzoli P, Checchi L, Ferri V, Landriani $S$, et al. Effectiveness of prophylactic antibiotics at placement of dental implants: a pragmatic multicentre placebo-controlled randomised clinical trial. Eur J Oral Implantol. 2010;3:135-143.

6. Esposito M, Cannizzaro G, Bozzoli P, Consolo U, Felice P, Ferri $\mathrm{V}$, et al. Efficacy of prophylactic antibiotics for dental implants: a multicentre placebo-controlled randomised clinical trial. Eur J Oral Implantol. 2008;1:23-31.

7. Arduino PG, Tirone F, Schiorlin E, Esposito M. Single preoperative dose of prophylactic amoxicillin versus a 2-day postoperative course in dental implant surgery: a two-centre randomised controlled trial. Eur J Oral Implant. 2015;8:143-149.

8. French D, Noroozi M, Shariati B, Larjava H. Clinical retrospective study of self-reported penicillin allergy on dental implant failures and infections. Quintessence Int. 2016;47:861-870.

9. Abu-Ta'a M, Quirynen M, Teughels W, Van Steenberghe D. Asepsis during periodontal surgery involving oral implants and the usefulness of peri-operative antibiotics: a prospective, randomized, controlled clinical trial. J Clin Periodontol. 2008;35:58-63.

10. Anitua E, Aguirre JJ, Gorosabel A, Barrio P, Errazquin JM, Román $\mathrm{P}$, et al. A multicentre placebo-controlled randomised clinical trial of antibiotic prophylaxis for placement of single dental implants. Eur J Oral Implantol. 2009;2:283-292.

11. Caiazzo A, Casavecchia P, Barone A, Brugnami F. A pilot study to determine the effectiveness of different amoxicillin regimens in implant surgery. J Oral Implantol. 2011;37:691-696.

12. Nolan R, Kemmoona M, Polyzois I, Claffey N. The influence of prophylactic antibiotic administration on post-operative morbidity in dental implant surgery. A prospective double blind randomized controlled clinical trial. Clin Oral Implants Res. 2014;25: 252-259.

13. Gristina AG, Hobgood CD, Webb LX, Myrvik QN. Adhesive colonization of biomaterials and antibiotic resistance. Biomaterials. 1987;8:423-426.

14. Gristina AG. Biomaterial-Centered Infections: microbial adhesion versus tissue integration. Science. 1987;237:1588-1595.

15. Roos-Jansåker AM, Lindahl C, Renvert H, Renvert S. Nine- to fourteen-year follow-up of implant treatment. Part I: implant loss and associations to various factors. J Clin Periodontol. 2006; 33:283-289.

16. Esposito M, Hirsch J-M, Lekholm U, Thomsen P. Biological factors contributing to failures of osseointegrated oral implants, (II). Etiopathogenesis. Eur J Oral Sci. 1998 Jun;106:721-764.

17. Figueiredo R, Camps-Font O, Valmaseda-Castellón E, GayEscoda C. Risk Factors for postoperative infections after dental implant placement: a case-control study. J Oral Maxillofac Surg. 2015;73:2312-2318.

18. von Elm E, Altman DG, Egger M, Pocock SJ, Gøtzsche PC, Vandenbroucke JP. The Strengthening the Reporting of Observational Studies in Epidemiology (STROBE) statement: guidelines for reporting observational studies. J Clin Epidemiol. 2008;61:344-349.

19. Maloney WJ, Weinberg MA. Implementation of the American Society of Anesthesiologists Physical Status classification system in periodontal practice. J Periodontol. 2008;79:1124-1126.

20. Hwang D, Wang HL. Medical contraindications to implant therapy: part I: absolute contraindications. Implant Dent. 2006;15:353-360.

21. Iacono V. Dental Implants in periodontal therapy. J Periodontol. 2000;71:1934-1942.

22. Krebs KA. Guidelines for the management of patients with periodontal diseases. J Periodontol. 2006;77:1607-1611.

23. Sawyer RG, Pruett TL. Wound infections. Surg Clin North Am. 1994;74:519-536.

24. Newson RB. Comparing the predictive powers of survival models using Harrell's C or Somers' D. Stata Jounal. 2010;10:339-358. 
25. Hayden JA, Côté P, Bombardier C. Evaluation of the quality of prognosis studies in systematic reviews. Ann Intern Med. 2006; 144:427-437.

26. Piattelli A, Piattelli M, Mangano C, Scarano A. A histologic evaluation of eight cases of failed dental implants: is bone overheating the most probable cause. Biomaterials. 1998;19:683-690.

27. Noack B, Genco RJ, Trevisan M, Grossi S, Zambon JJ, De Nardin E. Periodontal infections contribute to elevated systemic C-reactive protein level. J Periodontol. 2001;72:1221-1227.

28. Esposito M, Grusovin MG, Worthington HV. Interventions for replacing missing teeth: antibiotics at dental implant placement to prevent complications. Cochrane Database Syst Rev. 2013;7:CD004152.

29. Esposito M, Hirsch JM, Lekholm U, Thomsen P. Biological factors contributing to failures of osseointegrated oral implants. (I). Success criteria and epidemiology. Eur J Oral Sci. 1998;106:527-551.

30. Worthington P, Bolender CL, Taylor TD. The Swedish system of osseointegrated implants: problems and complications encountered during a 4-year trial period. Int J Oral Maxillofac Implants. 1987;2:77-84.

31. Zaugg LK, Astasov-Frauenhoffer M, Braissant O, Hauser-Gerspach I, Waltimo T, Zitzmann NU. Determinants of biofilm formation and cleanability of titanium surfaces. Clin Oral Implants Res. 2017;28:469-475.

32. Albouy JP, Abrahamsson I, Persson LG, Berglundh T. Implant surface characteristics influence the outcome of treatment of periimplantitis: an experimental study in dogs. J Clin Periodontol. 2011;38:58-64.

33. Rimondini L, Farè S, Brambilla E, Felloni A, Consonni C, Brossa $\mathrm{F}$, et al. The effect of surface roughness on early in vivo plaque colonization on titanium. J Periodontol. 1997;68:556-562.

34. Van Dyke TE, Sheilesh D. Risk factors for periodontitis. J Int Acad Periodontol. 2005;7:3-7.

35. Heitz-Mayfield LJ. Peri-implant diseases: diagnosis and risk indicators. J Clin Periodontol. 2008;35(Suppl 8):292-304.

36. Tran D, Gay I, Diaz-Rodriguez J, Parthasarathy K, Weltman R, Friedman L. Survival of Dental Implants Placed in Grafted and Nongrafted Bone: a Retrospective Study in a University Setting. Int J Oral Maxillofac Implants. 2016;31:310-317.

37. Rodriguez-Argueta OF, Figueiredo R, Valmaseda-Castellon E, Gay-Escoda C. Postoperative complications in smoking patients treated with implants: a retrospective study. J Oral Maxillofac Surg. 2011;69:2152-2157.

38. Chen H, Liu N, Xu X, Qu X, Lu E. Smoking, radiotherapy, diabetes and osteoporosis as risk factors for dental implant failure: a metaanalysis. PLoS One. 2013;8:71955.

39. Noda K, Arakawa H, Kimura-Ono A, Yamazaki S, Hara ES, Sonoyama $\mathrm{W}$, et al. A longitudinal retrospective study of the analysis of the risk factors of implant failure by the application of generalized estimating equations. J Prosthodont Res. 2015;59:178-184.

40. Javed F, Romanos GE. Impact of Diabetes Mellitus and Glycemic Control on the Osseointegration of Dental Implants: a Systematic Literature Review. J Periodontol. 2009;80:1719-1730.

41. Brocard D, Barthet P, Baysse E, Duffort JF, Eller P, Justumus $\mathrm{P}$, et al. A multicenter report on 1,022 consecutively placed ITI implants: a 7-year longitudinal study. Int J Oral Maxillofac Implants. 2000;15:691-700.

42. Kuula H, Könönen E, Lounatmaa K, Konttinen YT, Könönen M. Attachment of oral gram-negative anaerobic rods to a smooth titanium surface: an electron microscopy study. Int J Oral Maxillofac Implants. 2004;19:803-809.

43. Fürst MM, Salvi GE, Lang NP, Persson GR. Bacterial colonization immediately after installation on oral titanium implants. Clin Oral Implants Res. 2007;18:501-508.

44. Verdugo F, Laksmana T, Uribarri A. Systemic antibiotics and the risk of superinfection in peri-implantitis. Arch Oral Biol. 2016; 64:39-50.

\section{SUPPORTING INFORMATION}

Additional supporting information may be found online in the Supporting Information section at the end of the article.

How to cite this article: Camps-Font $\mathrm{O}$, MartínFatás P, Clé-Ovejero A, Figueiredo R, Gay-Escoda C, Valmaseda-Castellón E. Postoperative infections after dental implant placement: Variables associated with increased risk of failure. J Periodontol. 2018;89:11651173. https://doi.org/10.1002/JPER.18-0024 